\title{
Reexamination of the Carbohydrate Binding Stoichiometry of Lima Bean Lectin ${ }^{1,2}$
}

\author{
DAVID D. ROBERTS ${ }^{3}$ AND IRWIN J. GOLDSTEIN ${ }^{4}$
}

Department of Biological Chemistry, The University of Michigan, Ann Arbor, Michigan 48109

Received November 15, 1983

The carbohydrate binding stoichiometry of lima bean lectin component III was reexamined using equilibrium dialysis and quantitative affinity chromatography following limited chemical modification. Equilibrium dialysis employing methyl[2-14 C]benzamido2-deoxy- $\alpha$-D-galactopyranoside as ligand demonstrated that the lectin tetramer bound $4 \mathrm{~mol}$ of sugar with $K_{\text {assoc }}=1.44 \pm 0.13 \times 10^{3} \mathrm{M}^{-1}\left(\mathrm{~T}=5^{\circ} \mathrm{C}, \mathrm{pH} 7.0\right.$, ionic strength 0.1$)$. The previous report of two sites/tetramer [Bessler, W. and Goldstein, I. J. (1974) Arch. Biochem. Biophys. 165,444] appears to be the result of partial inactivation of the lectin due to oxidation of essential thiol groups. Following limited chemical modification of the thiol groups by methyl methanethiosulfonate, multiple intermediate forms with reduced affinity for Synsorb $A$ were obtained. The number and hemagglutinating activities of these intermediates provided further support for the presence of four carbohydrate binding sites on lima bean lectin component III.

The lima bean lectin was one of the first plant seed agglutinins shown to be blood group specific (1). Isolated in pure form by two groups (2-4), the lectin was shown to be a glycoprotein composed of 31-KDa subunits joined by a disulfide bridge to form dimers $\left(M_{\mathrm{r}}=62 \mathrm{KDa}\right)$. Two noncovalently linked dimers comprise lima bean lectin component III, and four such dimers afford lima bean lectin component II (2-4). The stoichiometry of carbohydrate binding was determined by equilibrium dialysis to be two sites/component III tetramer and four/component II octamer (5).

\footnotetext{
${ }^{1}$ This work was part of a dissertation submitted by $\boldsymbol{D}$. $\mathrm{U}$. Koberts in partial fulfillment of the requirements for the Doctor of Philosophy in the Horace $\mathbf{H}$. Rackham School of Graduate Studies at the University of Michigan.

${ }^{2}$ Dedicated to the Memory of Lee A. Murphy.

${ }^{3}$ Supported by National Institutes of Health Training Grant 5-T32-GM-07767 and a Rackham Predoctoral Fellowship from the University of Michigan.

${ }^{4}$ Supported by National Institutes of Health Grant GM-29470. To whom correspondence should be addressed.
}

Lima bean lectin (LBL) ${ }^{5}$ contains three classes of subunits (denoted $\alpha, \beta$ and $\alpha^{\prime}$ ) which can be resolved by isoelectric focusing under denaturing conditions (6). The subunit types have very similar but nonidentical peptide maps and amino acid compositions (6). At present there is no evidence for a difference in carbohydrate binding by the three subunit types.

LBL contains two cysteine residues/ subunit $(2,3)$. One cysteine in each subunit forms an intersubunit disulfide bond; the sulfhydryl group of the second cysteine was found to be required for carbohydrate binding activity (7-8).

The demonstration that haptenic sugars protected the free cysteine sulfhydryl groups present on each subunit against chemical modification by Ellman's reagent ruled out a model in which only one LBL subunit class bound carbohydrate (8). Other explanations consistent with the

\footnotetext{
${ }^{5}$ Abbreviations used: benzamido-MeaGalN, methyl2-benzamido-2-deoxy- $\alpha$-D-galactopyranoside; LBL, lima bean lectin; MMTS, methyl methanethiosulfonate; GalNAc, $\boldsymbol{N}$-acetylgalactosamine.
} 
data include negative cooperative binding of GalNAc or a binding site shared between two subunits. However, the requirement for activity of a sulfhydryl group which is labile to air oxidation (8) suggested that nonstoichiometric binding of GalNAc could also result from partial inactivation of the lectin during isolation or equilibrium dialysis. In this case, tetrameric LBL component III containing 1.0 free $\mathrm{SH} /$ subunit would be expected to bind $4 \mathrm{~mol}$ of GalNAc.

As a basis for further characterization of the sugar binding site of LBL and for understanding its action as a mitogen, the stoichiometry of sugar binding to LBL was reexamined. We repeated the determination of LBL component III stoichiometry by equilibrium dialysis using LBL containing 1.0 SH/subunit, and examined the effect of partial inactivation by limited thiol modification of the properties of LBL.

\section{MATERIALS AND METHODS}

Materials. Lima bean lectin was prepared from green lima beans (Phaseolus lunatus cv. Thorogreen) by affinity chromatography on Synsorb A (6). All buffers were degassed and saturated with $\mathrm{N}_{2}$ and maintained under a $\mathrm{N}_{2}$ atmosphere. Lectin prepared in this manner contained $1.06 \pm 0.03 \mathrm{SH} /$ subunit by titration with Ellman's reagent (9) and was stable to prolonged storage at $4^{\circ} \mathrm{C}$. Components II and III were isolated from the purified lectin by gel filtration on Ultrogel AcA-34 (6, 10). Methyl methanethiosulfonate (MMTS) was provided by Dr. J. Shafer (University of Michigan). Methyl 2-acetamido-2-deoxy- $\alpha$ D-galactopyranoside was prepared as described (11). $\left[{ }^{14} \mathrm{C}\right]$ Benzoyl chloride was prepared by refluxing $\left[{ }^{14} \mathrm{C}\right]$ benzoic acid (ICN, Irvine, Calif.) with a fivefold molar excess of thionyl chloride (Aldrich). The excess thionyl chloride was removed under vacuum (Caution: volatile radioactivity.) and the residual $\left[{ }^{14} \mathrm{C}\right] \mathrm{benzoyl}$ chloride used without further purification. Methyl [2${ }^{14}$ C] benzamido - 2 - deoxy - $\alpha$ - D - galactopyranoside (benzamido-Me $\alpha \mathrm{GalN}$ ) was prepared by reaction of a slight excess of $\left[{ }^{14} \mathrm{C}\right]$ benzoyl chloride with the amino sugar in methanol in the presence of $\mathrm{NaHCO}_{3}$. The product was recrystallized twice from methanol-ether and was of $>98 \%$ radiochemical purity ( $\mathrm{sp}$ act $1 \mathrm{Ci}$ / mol). The chromatographic and spectral properties of the labeled compound were identical to those of the unlabeled compound prepared as described elsewhere. ${ }^{6}$

\footnotetext{
${ }^{6}$ Kaifu, R. and Goldstein, I. J., submitted for publication.
}

Equilibrium dialysis. Binding of $\left[{ }^{14} \mathrm{C}\right]$ benzamido MeaGalN to LBL was conducted in microdialysis cells (Technilab Instruments, Inc., Pequannock, N. J.). Lectin and sugar were in $\mathrm{N}_{2}$-saturated sodium phosphate buffer, pH 7.0, ionic strength 0.1 . Following equilibration for $48 \mathrm{~h}$ with end-over-end mixing at $5^{\circ} \mathrm{C}$, the concentration of sugar in each chamber was determined by scintillation counting in ACS (Amersham). Lectin concentrations were determined by absorbance at $280 \mathrm{~nm}$ using $E_{1 \mathrm{~cm}}^{1 \%}=12.3(2)$.

Limited thiol modification. LBL was incubated under $\mathrm{N}_{2}$ with substoichiometric concentrations of MMTS for $24 \mathrm{~h}$. The reaction mixtures were then analyzed by affinity chromatography on a high-capacity Synsorb A column $(0.7 \times 14 \mathrm{~cm})$. The lectin was eluted using a $\mathrm{pH}$ gradient formed by running a linear gradient from buffer A (20 mM glycine, $20 \mathrm{mM}$ sodium formate, $20 \mathrm{mM}$ sodium succinate, $20 \mathrm{mM}$ sodium citrate, $0.1 \mathrm{mM} \mathrm{CaCl}_{2}, 0.1 \mathrm{mM} \mathrm{MnCl}_{2}, \mathrm{pH} \mathrm{6.8)} \mathrm{to} \mathrm{buffer}$ B (70 mM $\mathrm{HCl})$. The total gradient volume was 200 $\mathrm{ml}$ and was pumped at $77 \mathrm{ml} / \mathrm{h}$. Eluent was monitored at $280 \mathrm{~nm}$. Eluted lectin was dialyzed into sodium phosphate buffer, $\mathrm{pH}$ 7.0, ionic strength 0.1 for determination of thiol content. Activity was determined by hemagglutination of human Type $A_{1}$ red blood cells (4).

\section{RESULTS AND DISCUSSION}

The previous determination of the sugar binding stoichiometry of LBL by equilibrium dialysis employed $\left[{ }^{14} \mathrm{C}\right]$ methyl $\alpha$-DGalNAcp (5). This sugar was found to bind with relatively low affinity $\left(K_{\text {assoc }}=1 \times 10^{3}\right.$ $\mathrm{M}^{-1}$ at $2^{\circ} \mathrm{C}$ ). Since the $2-N$-benzamido analog bound with higher affinity (8) and could be prepared in radiolabeled form, it was used in place of methyl $\alpha-\mathrm{D}-\mathrm{GalNAc}$ in the present study. In order to ensure full activity of the lectin, the dialysis cells were flushed with nitrogen, and dialysis was limited to $48 \mathrm{~h}$. Thiol content was determined before and after dialysis to correct for any inactivation of lectin during dialysis. Specificity of the labeled ligand for the carbohydrate sites was confirmed by complete inhibition of binding in the presence of excess unlabeled methyl $\alpha-D^{-}$ GalNAc $p$.

A Scatchard plot (12) for binding of $\left[{ }^{14} \mathrm{C}\right]$ benzamido-Me $\alpha$ GalN to LBL component III (Fig. 1) indicated that fully active component III can bind $4 \mathrm{~mol}$ of carbohydrate $(n=1.07 \pm 0.06$ on a subunit basis assuming $M_{\mathrm{r}}=31,000$ ) with $K_{\text {assoc }}=1.44$ $\pm 0.13 \times 10^{3} \mathrm{M}^{-1}\left(\mathrm{~T}=5^{\circ} \mathrm{C}, \mathrm{pH} \mathrm{7.0, \text {ionic }}\right.$ 


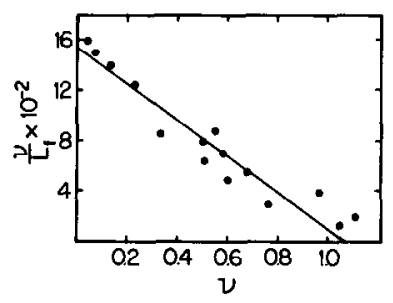

FIG. 1. Binding of $\left[{ }^{14} \mathrm{C}\right.$ benzamido-MeaGalN to lima bean lectin component III by equilibrium dialysis. Lima bean lectin (2-3 $\times 10^{-4} \mathrm{M}$ subunits) in sodium phosphate buffer, pH 7.0, ionic strength 0.1 was equilibrated with 0.025 to $5.7 \mathrm{mM}\left[{ }^{14} \mathrm{C}\right]$ benzamido-Me $\alpha \mathrm{GalN}$ at $5^{\circ} \mathrm{C}$ for $48 \mathrm{~h}$ under an $\mathrm{N}_{2}$ atmosphere. Sugar concentrations in each chamber were determined by counting quadruplicate $50-\mu \mathrm{l}$ aliquots. Stoichiometry of binding $(\nu)$ was calculated on a subunit basis using $M_{\mathrm{r}}=\mathbf{3 1 , 0 0 0}$.

strength 0.1). Determination of benzamidoMe $\alpha$ GalN binding by a thiol protection assay (8) under the same conditions $\left(\mathrm{T}=5^{\circ} \mathrm{C}\right.$, $\mathrm{pH} 7.0$, ionic strength 0.1 ) gave an apparent $K_{\text {assoc }}\left(K_{I}^{-1}\right)$ of $1.51 \times 10^{3} \mathrm{M}^{-1}$. Thus there is excellent agreement between the two methods for determining carbohydrate binding. The basis for the difference in stoichiometry from that reported previously is probably partial inactivation of the lectin prior to equilibrium dialysis. LBL isolated without precautions to minimize exposure to air typically contained 0.7 to $0.9 \mathrm{SH}$. Within 2 weeks the thiol content of purified lectin decreased to 0.5 to 0.6 $\mathrm{SH} /$ subunit. This instability was not seen with LBL prepared and stored under $\mathrm{N}_{2}$. The low binding affinity $\left(K_{\text {assoc }}=10^{3}\right)$ also probably contributed to the difficulty of obtaining an accurate value for the number of carbohydrate binding sites on the lectin.

Complete and reversible protection from chemical modification of a single free thiol group on each subunit of LBL by haptenic sugars is consistent with the stoichiometry determined using equilibrium dialysis. These results indicate the presence of a carbohydrate binding site on each subunit and a requirement of one thiol group for binding of each carbohydrate ligand. Therefore the measured stoichiometry should decrease linearly with modification of the thiols.
To confirm the sugar binding stoichiometry, we examined the properties of partially modified LBL. Affinity chromatography on Synsorb A was used to separate forms of LBL differing in valence. A similar approach has been utilized previously for separation of Griffonia simplicifolia isolectins $(13,14)$ and chemically modified concanavalin $A(15,16)$ on the basis of valence for an immobilized ligand. If LBL component III is divalent, partial modification should produce a monovalent derivative capable of being separated by affinity chromatography on Synsorb A. This intermediate would be inactive as a hemagglutinin. However, if component III is tetravalent, several intermediates which could be tri-, di-, and monovalent would be obtained. All except the latter intermediate would be expected to agglutinate erythrocytes. Chromatography of a natural mixture of LBL components on a Synsorb A column eluted with a $\mathrm{pH}$ gradient gave two peaks (Fig. 2a), representing components III and II as verified by electrophoresis at $\mathrm{pH} 4.3$ (17). Chromatography of the isolated components, prepared by gel filtration, gave single peaks on the Synsorb column (Fig. 2b) with the same retention times as observed in the mixture.

Treatment of purified component III with 0.5 equivalents of MMTS eliminated the component III peak. A broad, trailing peak followed by a peak at $80 \mathrm{~min}$ was obtained (Fig. 2c). The early, middle, and late portions of this profile were dialyzed against $\mathrm{pH} 6.8$ buffer and rechromatographed (Fig. 2d). Multiple peaks obtained in this experiment suggested that two to three distinct intermediates were present in partially modified LBL. The peak eluting at $80 \mathrm{~min}$ was stable to rechromatography and distinct from fully active component III. A second intermediate eluted as a broad peak at $30 \mathrm{~min}$, and the third was only weakly retarded by the column. All intermediates gave a single component III band on $\mathrm{pH} 4.3$ gel electrophoresis.

Following dialysis into sodium phosphate buffer, $\mathrm{pH} 7.0$, ionic strength 0.1 , thiol content and hemagglutination titers of the intermediates were determined (Table I). A progressive decrease in both thiol 


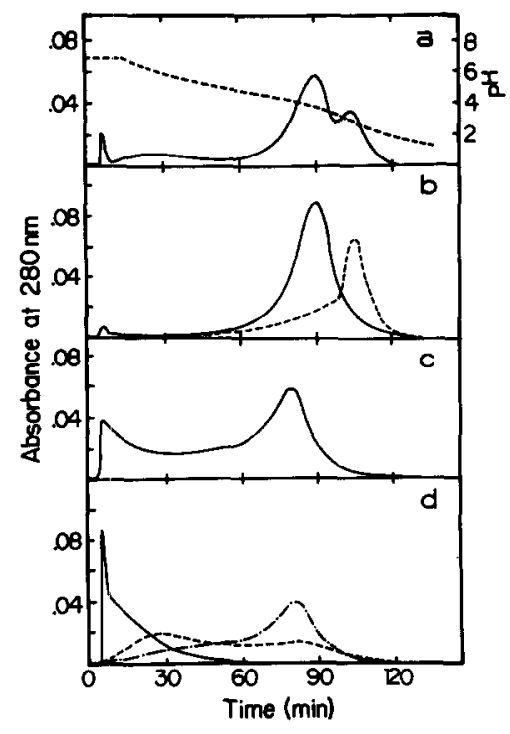

FIG. 2. Affinity chromatography of lima bean lectin on Synsorb A. Affinity chromatography was performed with a $\mathrm{pH}$ gradient elution as described under Materials and Methods. The $\mathrm{pH}$ gradient, presented in (a) was identical for all elution profiles. (a) $1.5 \mathrm{mg}$ natural mixture of lima bean lectin components; (b) LRL components isolated by gel filtration on Ultrogel AcA 34, (- $1.5 \mathrm{mg}$ component III, (- - $1 \mathrm{mg}$ component II; and (c) $1.5 \mathrm{mg}$ LBL component III modified with 0.5 equivalents of MMTS. (d) Pooled fractions from each region of the elution profile in (c) were dialyzed against $\mathrm{pH} 6.8$ buffer and reanalyzed: pool 1, 5-26 $\min$ (-); pool 2, 26-50 $\min (--4)$; pool $3,60-80 \mathrm{~min}(-\cdot-)$.

content and specific titer with earlier retention time demonstrated correlation between decreasing valence and increasing degree of modification. Weak hemagglutination activity in peak 1 would not be expected for monovalent LBL and probably results from contamination with divalent lectin.

The presence of multiple intermediates with hemagglutinating activity in partially modified LBL is consistent with the presence of four binding sites on component III, and confirms the stoichiometry determined by equilibrium dialysis. These results cannot be reconciled with a model wherein component III is divalent. The behavior of component II on affinity chromatography indicates that it has a higher valence than component III. Although it would be expected to be octavalent, this has not yet been confirmed by equilibrium dialysis.

Determination of the correct binding stoichiometry is crucial for interpreting the effects of lectins on cells. The affinity of lectin binding to cell surface carbohydrates and the mitogenic effect of lectins on lymphocytes are dependent on lectin valence. Derivatives of concanavalin A with reduced valence have been prepared and found to differ in their mitogenic properties from the native tetravalent lectin $(15,18)$.

The effect of valence on mitogenicity of LBL has also been studied. LBL component III, previously reported to be divalent (5), was a very weak mitogen $(19,20)$. Component II, having twice the valence of component III, was a potent mitogen. Natural polymers of LBL (21) or chemically crosslinked component III (22) also displayed enhanced potency as mitogens. Chemical crosslinkage of native soybean agglutinin into dimers and higher oligomers greatly enhanced its hemagglutinating and lymphocyte-transforming activities (23). Lyophilization of the soybean lectin also enhanced its mitogenic activity (24). The present demonstration that LBL component III has four binding sites warrants its reexamination as a mitogen under conditions which protect its thiol groups from oxidation.

In conclusion, the results of two independent methods are consistent with LBL

TABLE I

Hemagglutinating Activity and Thiol Content of MoDIFIEd Lima Bean Lectin

\begin{tabular}{lcc}
\hline \multicolumn{1}{c}{ Lectin } & $\begin{array}{c}\text { Specific titer } \\
\left(\mathrm{mg} \mathrm{m}^{-1}\right)\end{array}$ & $\begin{array}{c}\text { SH/ } \\
\text { subunit }\end{array}$ \\
\hline $\begin{array}{l}\text { Component II } \\
\text { Component III }\end{array}$ & 3700 & 1.0 \\
$\begin{array}{l}\text { Modified component } \\
\text { III-pool 1 }\end{array}$ & 420 & 1.0 \\
$\begin{array}{l}\text { Modified component } \\
\text { III-pool 2 }\end{array}$ & 5 & 0.26 \\
$\begin{array}{l}\text { Modified component } \\
\text { III-pool 3 }\end{array}$ & 30 & 0.37 \\
\hline
\end{tabular}


component III being tetravalent for carbohydrate. In addition to defining the stoichiometry of LBL, the procedures presented here provide a method for preparing LBL component III with a variable number of carbohydrate binding sites. These forms will be of interest in further examining the effects of valence on the biological properties of LBL.

\section{ACKNOWLEDGMENT}

We thank Garth Goldstein for preparing the lima bean meal for this study.

\section{REFERENCES}

1. BoYd, W. C., AND REQUERA, R. M. (1949) J. Immunol 62, 333-339.

2. Gould, N. R., AND ScheINBERG, S. L. (1970) Arch. Biochem. Biophys. 137, 1-11.

3. Galbraith, W., AND GoldSTEIN, I. J. (1970) FEBS Lett. 9, 197-201.

4. Galbraith, W., AND Goldstein, I. J. (1972) Biochemistry 11, 3976-3984.

5. Bessler, W., AND Goldstein, I. J. (1974) Arch. Biochem. Biophys. 165, 444-445.

6. RoBerts, D. D., ETZLER, M. E., AND GoLDSTEIN, I. J. (1982) J. Biol. Chem. 257, 9198-9204.

7. Gould, N. R., AND SCHEINBERG, S. L. (1970) Arch. Biochem. Biophys. 141, 607-613.

8. RoBERTS, D. D., AND GoldsteIN, I. J. (1984) $J$. Biol. Chem. 259, 903-908.

9. Ellman, G. L. (1959) Arch. Biochem. Biophys. 82, 70-77.
10. Pandolfino, E. R., and Magnuson, J. A. (1980) J. Biol. Chem. 255, 870-873.

11. Buss, D. H., AND Goldstern, I. J. (1968) J. Chem. Soc. $c, 1457-1461$.

12. SCATChard, G. (1949) Ann. N. Y. Acad Sci. 51, 660-672.

13. MURPHY, L. A., AND GoldSTEEIN, I. J. (1977) J. Bioh Chem. 252, 4739-4742.

14. Delmotte, F. M., AND Goldstein, I. J. (1980) Eur. J. Biochem. 112, 219-223.

15. Fraser, A. R., Hemperly, J. J., Wang, J. L., AND Edelman, G. M. (1976) Proc. Nath Acad. Sci. USA 73, 790-794.

16. Beppu, M., Terao, T., and OSaWA, T. (1975) $J$. Biochem. 78, 1013-1019.

17. Reisfeld, R., Lewis, U. J., AND Williams, D. E. (1962) Nature (London) 195, 281-283.

18. Saito, M., TakakU, F., Hayashi, M., Tanaka, I., ABE, Y., NAGAI, Y., AND ISHII, S. (1983) J. Biol. Chem. 258, 7499-7505.

19. RudDon, R. W., Weisenthal, L. M., Lundeen, D. E., BESSLER, W., AND GolDSTEIN, I. J. (1974) Proc. Natl Acad. Sci. USA 71, 1848-1851.

20. Bessler, W., Resch, K., AND Ferber, E. (1976) Biochem. Biophys. Res. Commun 69, 578-585.

21. KraUt, H., ResCH, K., AND BeSSLER, W. G. (1979) in Protides of Biological Fluids (Peeters, H., ed.), pp. 619-622, Pergamon, Oxford.

22. MuNSKe, G. R., PANDOLFINO, E. R., AND MaGNuSON, J. A. (1981) $J$. Immunol 127, 1607-1610.

23. LotAN, R., LIS, H., ROSENWASSER, A., NovogroDSKY, A., AND Sharon, N. (1973) Biochem. Biophys. Res. Commun. 55, 1347-1355.

24. SHECHTER, B., LIS, H., LOTAN, R., NOVOGRODSKY, A., AND SHARON, N. (1976) Eur. J. Immunol. 6, 145-149. 